\title{
Applications magnétoélectriques des supraconducteurs
}

Cet ouvrage est un des titres du secteur Sciences de la matière de la Collection Grenoble Sciences d'EDP Sciences, qui regroupe des projets originaux et de qualité. Cette collection est dirigée par Jean Bornarel, Professeur émérite à l’Université Grenoble Alpes.

\section{Comité de lecture de l'ouvrage}

- Julien Bobroff, Professeur à l'Univ. Paris-Sud, Lab. de physique des solides - Orsay

- Claude Bourbonnais, Professeur titulaire à l'Univ. de Sherbrooke, dép. de physique Canada

- Daniel Bourgault, Directeur de recherche au CNRS, Inst. Néel - Grenoble

- Hervé Courtois, Professeur à l'Université Grenoble Alpes, Inst. Néel - Grenoble

- Jean-Luc Duchateau, Conseiller scientifique au CEA, Inst. de Recherche sur la Fusion par confinement magnétique - Cadarache

- Pascal Febvre, Professeur à l'Univ. de Savoie, Inst. de Microélectronique Électromagnétisme et Photonique - Le Bourget du Lac

- Claire Hérold, Directeur de recherche au CNRS, Inst. J. Lamour - Vandoeuvre-lèsNancy

- Thierry Klein, Professeur à l’Univ. Grenoble Alpes, Inst. Néel - Grenoble

- Jean-Pierre Michel, Professeur émérite à l'École des Mines de Nancy

- Alessandro Monfardini, Chargé de recherche au CNRS, Inst. Néel - Grenoble

- Lucio Rossi, Professeur à l'Univ. de Milan, chef du projet High Luminosity LHC au CERN - Suisse

- Pierre Rodière, Chargé de recherche au CNRS, Inst. Néel - Grenoble

- André Sulpice, Directeur de recherche au CNRS, Inst. Néel - Grenoble

- Jean-Louis Tholence, Directeur de recherche émérite au CNRS, Lab. National des Champs Magnétiques Intenses - Grenoble

- Pierre Vedrine, Ingénieur au CEA, Inst. de recherche sur les lois fondamentales de l'Univers - Saclay

- Georges Waysand, Directeur de recherche au CNRS, Lab. souterrain à bas bruit - Rustrel.

Cet ouvrage a été suivi par Laura Capolo, Gwenn Cognard \& Anne-Laure Passavant pour la partie scientifique et la réalisation pratique (avec la participation de Patrick Dessenne et Anne-Claire Lecomte pour les figures et photographies).

\section{Ouvrages labellisés sur des thèmes proches (chez le même éditeur)}

Supraconductivité - Introduction (P. Mangin \& R. Kahn) - Matériaux supraconducteurs Structures et propriétés physico-chimiques (P. Mangin \& R. Kahn) - Applications des supraconducteurs en couches minces - SQUIDs, Détecteurs, Électroniques (P. Mangin \& R. Kahn) - Physique des diélectriques (D. Gignoux \& J.-C. Peuzin) - Magnétisme - Fondements et Matériaux et applications (sous la direction d'E. du Trémolet de Lacheisserie) 